\title{
A gas adsorption porosimetry analysis of Portland cement prepared by compaction vs. compaction with indirect ultrasonic agitation
}

\author{
Nader Azizi ${ }^{1}$, Yltze P. Cubas ${ }^{2}$, Maria F. Orellana ${ }^{3}$ \\ ${ }^{1} D D S, M S c$, Private Practice, New York City, USA \\ ${ }^{2} D D S$. Post-doctoral fellow, Department of Orofacial Sciences, School of Dentistry, University of California, San Francisco, USA \\ ${ }^{3} D D S, M S c$, PhD. Director Predoctoral Orthodontic Program, Department of Orofacial Sciences, Division of Orthodontics, School of Dentistry, University of California, \\ San Francisco, USA
}

\section{Abstract}

Background: Mineral trioxide aggregate (MTA), is commonly used in endodontic and restorative procedures. Objective: Our objective was to introduce gas adsorption porosimetry as a viable method for evaluation of general porosity and specific pore characteristics of set Portland cement used in substitute for MTA, to investigate the effect of two different obturation methods (compaction and compaction in conjunction with indirect ultrasonic agitation of the cement paste), and to evaluate the correlation between the specific pore characteristics to compressive strength in general. Material and Methods: Portland cement samples were prepared and divided into two groups based on compaction techniques. An initial stereomicroscopic evaluation was done to assess any differences in appearance of pores randomly selected from either of the two experimental groups. Specific pore characteristics and compressive strength werequantified by a gas adsorption porosimeter and an Instron universal testing machine. A two-tailed student t-test was used for statistical comparison of data, and a regression analysis was done to evaluate the correlation between each specific pore characteristic and compressive strength in general. Results: The gas porosimetry method provided measurable values relating to specific pore characteristics of Portland cement. The stereomicroscopy evaluation revealed marked differences between samples from the two groups, namely visibly larger pores both on the outside surface and in cross-sections of specimens prepared by the indirect ultrasonic activation method. Conclusions: Gas adsorption porosimetry is a feasible method for evaluation of specific pore characteristics of Portland cement and potentially other dental materials as well.

\section{Introduction}

The key requirement for the strength and stability of hydraulic cement is that the hydrates formed on immediate reaction with water should be essentially insoluble in water. Mineral Trioxide Aggregate (MTA) is an inorganic hydraulic cement used as a restorative material in dentistry. Portland cement is reported to be the main component of this inorganic hydraulic cement. Similar to other inorganic cements, MTA can set and harden in moist environments [1], a property which makes it ideal for use in dentistry. In addition, MTA has been shown to provide a predictable seal against leakage in bacterial and dye leakage studies [2-4]. The calcium hydroxide com- ponent released during its hydration reaction provides alkalinity to this material, which may account for both its antibacterial and hard-tissue growth induction properties $[5,6]$

MTA placement technique affects some of the structural and material properties of the set cement $[7,8]$. These properties range from the cement's adaptation to its container's walls, to changes in its resistance to leakage and variations in its surface and internal porosity. It is now well accepted that pores decrease the mechanical properties of cement [9]. Therefore, any technique that would potentially affect the porosity of MTA may similarly affect its mechanical properties. Selection of handling and placement methods, which optimize the
Citation: Azizi N, Cubas Y, Orellana M. (2014) A gas adsorption porosimetry analysis of Portland cement prepared by compaction vs. compaction with indirect ultrasonic agitation. Dentistry 3000. 1:a001 doi:10.5195/d3000.2014.24

Received: March 25, 2014

Accepted: October 15, 2014

Published: November 3, 2014

Copyright: (C2014 Azizi et al. This is an open access article licensed under a Creative Commons Attribution Work 4.0 United States License.

Email: OrellanaM@dentistry.ucsf.edu

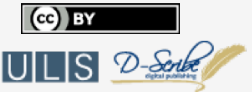

New articles in this journal are licensed under a Creative Commons Attribution 4.0 United States License.

This journal is published by the University Library System, University of Pittsburgh as part of its D-Scribe Digital Publishing Program and is cosponored by the University of Pittsburgh Press. physical and mechanical properties of the cement, may prove beneficial. For instance, commonly observed flexural weakness of cements has been related to presence of large voids in a set material. The removal of macro-defects during preparation has been shown to increase the flexural strength of hydraulic cements [10]. Similar effects may potentially be expected in respect to compressive strength.

Pores in hydraulic cements, such as MTA, may stem from a number of sources. They could be either internal in the source, such as those created as a result of the material's hydration reaction, or they could have an external source. Internal sources of porosity may have a profound impact on its mechanical properties as 
powder-to-water is the most important parameter influencing the porosity of hydraulic cements [11]. Selection of the powder-to-water ratio would affect the porosity of a cement mix due to its effect on the size and distribution of its capillary-pores which are inevitably formed during the hydration reactions. Similarly, external sources of porosity may also influence some structural properties of restorative cements. For example, direct ultrasonic activation of an MTA cement paste during its placement has been linked to entrapment of larger sized air-bubbles in the set specimen [12] possibly due to a proposed "whipping" motion of the ultrasonic delivery device. These larger pores, similar to those affecting the flexural strength of the material, may affect on the compressive strength of the material.

Although MTA is traditionally used in low pressure bearing areas, its use in high pressure bearing areas may potentially be indicated (i.e. as bases under other restorative dental materials). Some have proposed the use of Portland cement as an inexpensive alternative to other more expensive dental restorative materials for restoration of coronal defects [13], with potential uses in economically challenged areas. When used in such critical locations, both MTA and Portland cement must withstand compressive forces that could challenge their clinical longevity through time. Other factors affecting porosity such as pore size distributions, microcracks, interface, among others are also important in determining the mechanical properties of cement materials $[14,15]$. However, porosity which can be semi-empirically and concisely used to describe the relationship between strength and microstructure of porous material is still being studied [16, 17]. Porosity has been shown to display an inverse relationship to compressive strength, with reduction of porosity leading to increases in compressive strength of a solid but porous material.

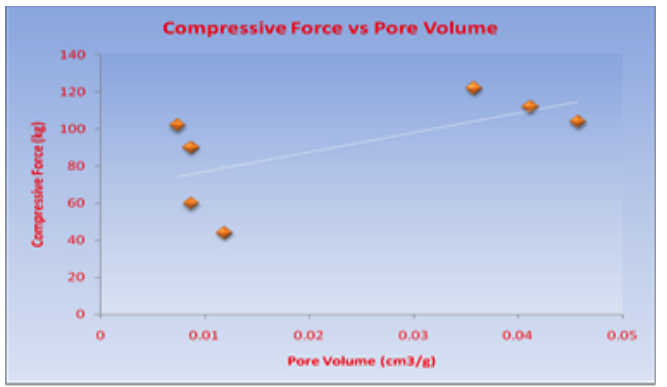

a
Gas adsorption has been one of the most popular techniques used for the study of pore structure in materials that contain micropores and mesopores, or mainly pores in cement pastes with radii between $1 \mathrm{~nm}$ and approximately $60 \mathrm{~nm}$. Therefore, gas adsorption technique can mainly characterize gel pores and small and medium capillary pores. Gas adsorption methods are based on measurements of the amount of gas adsorbed on the surface of a powder on the monomolecular depth of the particle's surface [18]. When a porous solid is exposed to gas of a certain volume and pressure, it begins to adsorb the gas molecules on its outside surface and inside its pores. The amount of gas adsorbed during an adsorption experiment can then be calculated by either a gravimeric, volumetric, or thermal conductivity measurement method. From the amount of gas adsorbed and the corresponding relative pressure recorded during the experiment, a great number of methods are developed for analysis which include methods for determining the pore volume, pore size distribution, and the Brunauer-Emmett-Teller (BET) specific surface area of the material. Surface area measurements are the most widely used means for characterization of porous materials. Since the surface corresponds to the roughness of the particle and its porous interior, gas adsorption is the preferred technique $[19,20]$.

We investigated the effect of two common clinically practiced placement techniques on porosity and compressive strength of Portland cement in an in vitro model. We compared the porosity of samples of Portland cement placed by either compaction alone (Group 1) or compaction in conjunction with indirect ultrasonic activation of the cement paste during its placement (Group 2). Initially, stereomicroscopic inspections of samples were made in an attempt to make visual comparisons between specimens selected from either of the

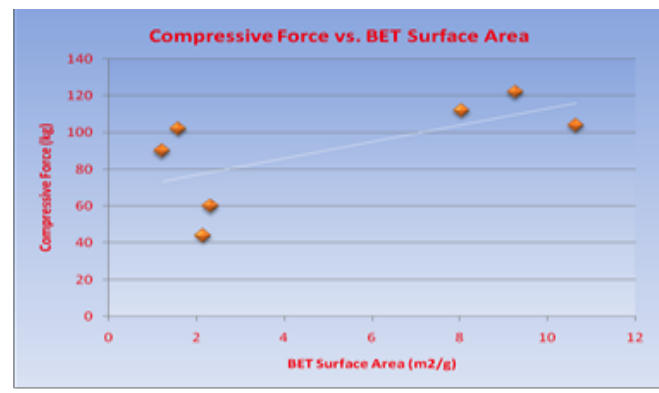

b two experimental groups. Later, a gas adsorption porosity method was used to quantify and compare few specific pore characteristics of the cement samples - namely their BET specific pore surface area, pore volume, and pore size distribution- from both groups. Compressive strengths of samples were quantified using an Instron universal material testing machine.

\section{Materials and Methods}

In a clean glass dappen dish, $1.0 \mathrm{~g}$ of Portland cement was mixed with $0.32 \mathrm{ml}$ of deionized water using a metal mixing spatula. Care was taken to gently mix the two components to avoid introduction of large air bubbles into the cement paste. Mixing was done for 30 seconds until the material took a consistency which allowed for easy transfer and placement of its aliquots by an amalgam carrier into metallic moulds. Then each sample followed treatment by either of the two methods of obturation. In Group 1, 8 samples were obturated into the molds using a ten second application of an equal and constant amount of vertical force to the cement paste. A five pound weight placed on top of a piston connected to the metallic obturator delivered the vertical component of the obturation force. In Group 2, 8 samples were prepared similarly, with the difference of indirect ultrasonic energy. The tip of a vibrating ultrasonic instrument (BUC-2) was placed along an area about one inch above the tip of the obturator to transfer a 10 second burst of ultrasonic energy to the metallic instrument. The obturator, in turn, propagated the ultrasonic energy to the paste along with delivering its vertical component force of compaction. A gridded plastic separator was placed over a thin pool of water at the bottom of the container with the molds placed on top. The plastic separator was used to prevent the samples from directly coming in contact with the water reservoir, while allowing them to set at nearly $100 \%$

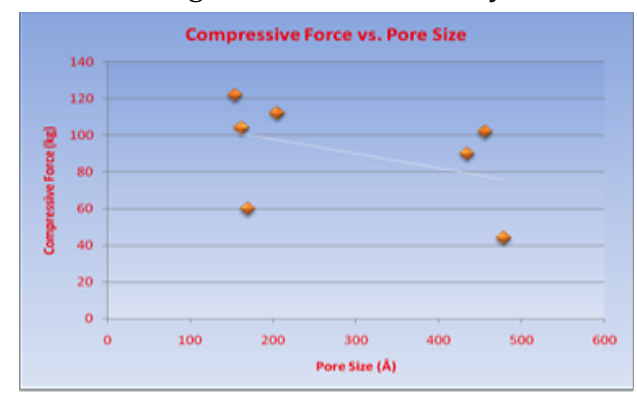

C

Figure 1: Best fit lines through intercepts of specific pore characteristics and compressive strength of samples. a,b. Graphs show a slight positive correlation. c. Graph shows a slight negative correlation. 
humidity and at room temperature. After one week, setting of samples was visually confirmed. Samples were then pushed out of each mold and prepared for porosity and compressive strength evaluations. Randomly selected samples from each group were prepared for stereomicroscopic evaluation (5X, 10X, and 20X magnifications.) The external surfaces of samples were initially evaluated for presence of visibly large surface pores. Then, samples were ground down to make half-cylinders of samples and exposing their internal appearance.

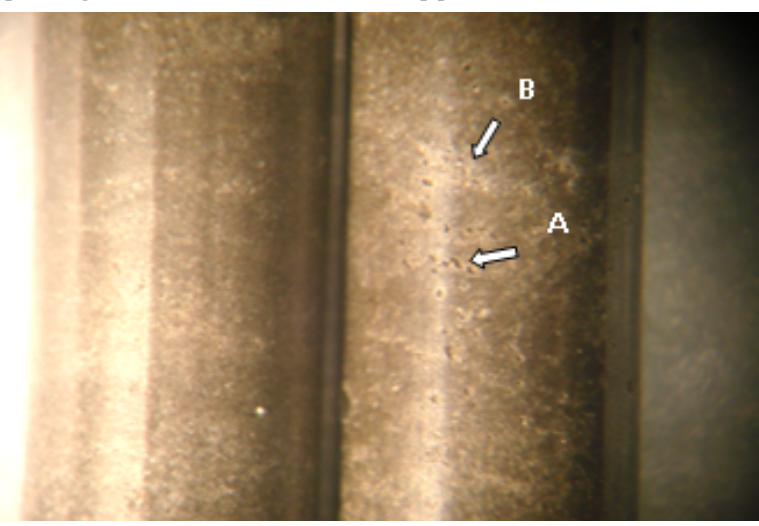

Figure 2: Samples of set cement placed side by side under $5 \mathrm{x}$ magnification. Note the presence of visible pores on the surface of specimen from the indirect ultrasonic activation group (A); Slight variation in color marks the

Stereomicroscopic evaluations of internal surfaces were also done for presence of notably visible pores.

\section{Gas adsorption porosimetry evaluation}

Samples were sent to Micrometrics (Micromeritics, Norfolk, GA, USA.) and evaluated after varying durations of setting time. Specimens were de-gassed at $40^{\circ} \mathrm{C}$ for 16 hours then placed in a sample tube and heated under vacuum or flowing gas to remove contaminants on the surfaces of the samples. The sample tube was then placed in the analysis port of a 2420 Accelerated Area and Porosimetry System for automatic analysis. The krypton adsorption isotherm was recorded at $120 \mathrm{~K}$.

\section{Compressive strength analysis}

Cylindrical samples from each experimental group were carefully placed on their vertical axes to stand perpendicular to the two horizontal plates of the Instron testing machine. The mobile upper plate was set to advance at a uniform speed of $1 \mathrm{~mm}$ per second to deliver the crushing vertical forces to the samples. Increasing vertical forces were applied to each sample until structural failure was achieved, at which point this maximum weight was recorded.

\section{Statistical Analsysis}

\section{Results}

\section{pore surface} Pore volume 1b).

Statistical analysis comparing the average values of specific pore characteristics and compressive strength of samples was done using a two-tailed student's t-test. Correlation and regression tests were used to look for any relationship between each specific pore characteristic (BET specific surface area of pores, pore volume, and pore size distribution) and compressive strength in general.

In Table 1, we observed an apparent trend in BET pore surface area and a trend in pore volume of samples in Group 1, however; the difference in values are not statistically significant.

Additionally, there is an apparent trend in pore size and a trend in compressive strength of samples in Group 2, but the difference in values is not statistically significant.

\section{Regression and Correla- tion Analysis Compressive strength vs. BET specific}

This revealed a positive regression with a correlation coefficient of +0.65 . This shows that as the BET pore surface area of samples increased, so did the compressive strength of the samples (Figure 1a).

Compressive strength vs.

A positive regression with a correlation coefficient of +0.64 shows that if the pore volume of samples increased, so did the compressive strength of the samples (Figure

\section{Compressive strength vs. Pore size dis- tribution}

A negative regression with a correlation coefficient of -0.42 shows that if the pore size of samples increased, the compressive strength of samples tends to decrease (Figure 1c).

\section{Stereomicroscopy}

$5 \mathrm{X}$ magnifications of randomly selected samples from each group were placed side by side. Initially we noticed visible differences in the surface appearance of the two cylindrical specimens. The sample prepared by vertical compaction force alone presented a more uniform appearance of its outside surface area with no obvious large porosities or transition lines. However, this specimen presents numerous larger porosities and a number of transition lines which correspond to the junction between aliquots of cement paste place on top of each other during the segmental compaction of the cement.

The same side by side comparison of cross sections of samples were arranged as before, but at 10X magnification reveals similar arrangement of larger pore sizes and transition lines present throughout the interior bulk of the sample prepared by compaction in conjunction with ultrasonic activation (Figure 3a). A closer look at transition lines visualized at $25 \mathrm{X}$ magnification reveals weak faults created between aliquots of cement samples placed by the combined compaction and ultrasonic activation method (Figure 3b).

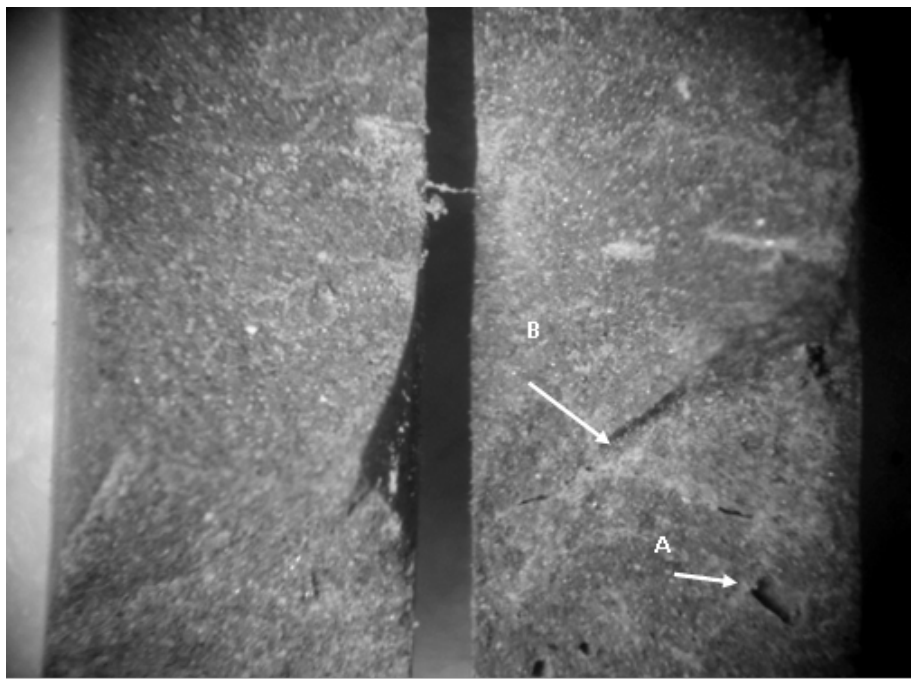

Figure 3: At 10x magnification, large internal pores (air-bubbles) could be seen in cross sections of samples from the indirect ultrasonic activation group (A); Transition areas between aliquots of cement present as "watered-down, weak fault-lines.

\section{Discussion}

In this study, we introduced gas adsorption porosimetry as an acceptable method for evaluation and distinction of specific pore characteristics of a dental restorative material, namely its BET specific surface area, pore volume and pore size distribution. This method of analysis generates quantitative data with values that reflect on those specific pore characteristics of solid but porous materials. According to 
Ying-zi et al "gas adsorption porosimetry is suitable for the materials with apertures smaller than $50 \mathrm{~nm}$ or the powder with the particle size smaller than 100nm [20]." Our data showed that gas adsorption porosimetry could be used for comparative evaluation and statistical analysis of pore characteristics of different dental materials in addition to evaluating the effects of chemical or physical manipulations on the materials' specific pore characteristics. Lee at al stated that gas adsorption technique gives the largest amount of information for the porous structure of solids [21].

When comparing the pore characteristics of samples placed by either of the two tested placement methods, our overall preliminary results show no significant differences between the BET pore surface area, pore volume, and pore size distribution of samples from either of the two groups however; these data displayed a number of observed trends which indicate a positive effect on compaction in conjunction with indirect ultrasonic technique. The numbers of specimens tested in this study were limited due to the high cost of a larger scale analysis.

Based on the limited number of samples tested, eight samples per group, no statistically significant differences in either the average BET specific surface area, pore volume or pore size distribution of samples from either of the two groups may be reported. However, from this data trends may be observed. First, our data showed a trend toward larger average surface area of pores in those samples prepared by compaction alone. In contrast, there was a trend toward smaller average surface area of pores in those samples prepared by compaction in conjunction with ultrasonic activation of the paste during its placement. Second, we observed similar results with samples prepared by compaction alone showing a trend toward larger average pore volumes compared to those prepared by the indirect ultrasonic activation method. Lastly, this data revealed a trend which showed smaller average pore size distributions in samples prepared by compaction compared to those made using the indirect ultrasonic activation method.

Surface and cross sectional stereomicroscopic inspection of the samples from each group led us to a number of initial impressions related to structural differences between the two. It appeared that a sample prepared by compaction alone had a more uniform outer surface which was devoid of any noticeably large sized airentrapped pores that were more readily observed on the outer surface of the sample prepared by the indirect ultrasonic activa- tion of the paste during its placement. A similar pattern was also observed in midline cross sectional preparations of samples from both groups. These cross sectional preparations also revealed the presence of more numerous air-entrapped pores in samples prepared by ultrasonic activation. Similar observations have also been reported in other investigations where a "direct" activation of the cement through insertion of the vibrating ultrasonic tip into the paste is thought to create a "whipping" motion in the paste, leading to introduction of larger air bubbles into the paste [14]. Another explanation for this observation may be related to a phenomenon of the action of ultrasonically vibrating instruments in liquid media, call "acoustic cavitation [22]". This phenomenon may potentially introduce larger bubbles into a more viscous medium, such as that of our Portland cement paste specimens.

Another noteworthy observation that was made only in the sample prepared by the indirect ultrasonic activation was the presence of horizontal demarcation lines between aliquots of cement placed during obturation of the mold. A plausible explanation for formation of such demarcations may be that the agitation provided by the ultrasonic energy may have potentially forced the solid particles of Portland cement to stack-up more closely against each other by forcing any unbound water out of the spaces between those particles, and essentially, causing sedimentation of the solid entities in the water-cement paste mixture. Other reports have shown that a critical increase in the liquid component of hydraulic cement would lead to a decrease in the strength of the material. These "watered-down" interfaces may ultimately present as those weak "fault-lines" seen in the indirect ultrasonically prepared samples in our magnified images.

The presence of visually larger pores in samples placed by the indirect ultrasonic activation method concur with our report of larger values for average pore sizes along with the expected correspondingly smaller average pore volume and average pore surface area of samples prepared with the aid of an indirect ultrasonic activation method. Such findings are in contrast to possibly more sedimentation of solid Portland cement particles in the paste prepared by the indirect ultrasonic method. This conclusion was based on the pooling of water observed on top of each aliquot of cement paste placed to make up a whole sample. This would suggest that sa

mples prepared by the indirect ultrasonic activation method would expectedly have smaller pores sizes, while presenting larger pore surface areas and pore volumes. Yet this was not supported by our quantitative data. We believe that the indirect ultrasonic

Table 1: Average values of group 1 and group 2 .

\begin{tabular}{|c|c|c|c|}
\hline & Group 1 & Group 2 & p-value \\
\hline $\begin{array}{l}\text { BET Specif- } \\
\text { ic Pore Sur- } \\
\text { face }\end{array}$ & $\begin{array}{c}6.175 \mathrm{~m}^{2} / \mathrm{g} \\
\mathrm{SD}=3.0\end{array}$ & $\begin{array}{l}5.172 \mathrm{~m}^{2} / g \\
\mathrm{SD}=4.072\end{array}$ & 0.762 \\
\hline $\begin{array}{l}\text { Pore Vol- } \\
\text { ume }\end{array}$ & $\begin{array}{c}0.0257 \mathrm{~m}^{3} / \mathrm{g} \\
\mathrm{SD}=0.02\end{array}$ & $\begin{array}{c}0.0241 \mathrm{~m}^{3} / \mathrm{g} \\
\mathrm{SD}=4.072\end{array}$ & 0.922 \\
\hline $\begin{array}{l}\text { Pore Size } \\
\text { Distribu- } \\
\quad \text { tion }\end{array}$ & $\begin{array}{l}239.886 \AA \\
S D=144.27\end{array}$ & $\begin{array}{c}318.09 \AA \\
\mathrm{SD}=162.27\end{array}$ & 0.4984 \\
\hline $\begin{array}{l}\text { Compres- } \\
\text { sive } \\
\text { Strength }\end{array}$ & $\begin{array}{c}88.667 \mathrm{~kg} \\
\mathrm{SD}=24.8\end{array}$ & $\begin{array}{c}92.0 \mathrm{~kg} \\
\mathrm{SD}=34.7\end{array}$ & 0.894 \\
\hline
\end{tabular}

activation creates more densely packed specimens with smaller pores and potentially larger pore surface areas and pore volumes. The larger air bubbles, as visualized in stereomicroscopic evaluations, may be outliers that skew the data enough to support results reporting larger average pore size distributions and smaller average pore surface areas and pore volumes that contradict the visually observed signs of sedimentation in the indirectly activated ultrasonic method. Excluding these outliers we report that samples prepared by indirect ultrasonic activation are indeed more densely packed at a more microstructural level when compared to those placed by compaction alone. Such extrapolations may be further supported when comparisons between data reflecting the average compressive strength of samples from the two groups is made.

Our data showed that the placement method did not have a statistically significant effect on the average compressive strength of the samples from the two groups; however, there was a trend toward samples in the ultrasonically activated group having a higher average compressive strength values than those samples placed 
by compaction alone. Presence of few airbubbles may be compensated by the more densely packed cement particles at a microstructural level in the indirect ultrasonic method. This density may be related to the observed higher average compressive strength of samples from this group.

\section{Conclusions}

Gas adsorption porosimetry is a method that can be potentially used for evaluation of specific pore characteristics of dental restorative materials. Larger scale studies are needed to evaluate the superiority of one method of placement over the other.

\section{References}

1. Evaluation of setting properties and retention characteristics of mineral trioxide aggregate when used as a furcation perforation repair material. Sluyk SR, Moon PC, Hartwell GR. J Endod. 1998 Nov;24(11):768-71. PMID: 9855831.

2. A comparison of MTA, Super-EBA, composite and amalgam as root-end filling materials using a bacterial microleakage model. Adamo, HL, Buruiana $\mathrm{R}$, Schertzer L, Boyland RJ. IntEndod J. 1999 May;32(3):197-203. PMID: 10530207.

3. Leakage evaluation of root end filling materials using endotoxin. Tang HM, Torabinejad M, Kettering JD. J Endod. 2002 Jan;28(1):5-7. PMID: 11806652.

4. Dye leakage of four root end filling materials: effects of blood contamination. Torabinejad M, Higa RK, McKendry DJ, Pitt Ford TR.J Endod. 1994 Apr;20(4):159-63. PMID: 8035153.

5. Antibacterialeffects of some rootendfillingmaterials. Torabinejad $M$, Hong CU, Pitt Ford TR, Kettering JD. J Endod. 1995 Aug;21(8):403-6. PMID: 7595152.

6. Reaction of rat connective tissue to implanted dentin tubes filled with a white mineral trioxide aggregate. Holland R1, Souza Vd, Nery MJ, FaracoJúnior IM, Bernabé PF, OtoboniFilho JA, DezanJúnior E. Braz Dent J. 2002;13(1):23-6. PMID: 11870957.

7. A comparison of laterallycondensedgutta-percha, thermoplasticizedgutta-percha, and mineral trioxide aggregate as root canal filling materials. Bogen G. J Endod. 2004 Dec;30(12):826. PMID: 15564859.

8. A quantitative comparison of the fill density of MTA produced by two placement techniques. Yeung P1, Liewehr FR, Moon PC. J Endod. 2006 May;32(5):456-9. PMID: 16631848.

9. Porosity-dependence of effec

10. tive mechanical properties of poresolid composite materials. Pal R. J Com-pos Mater. 2005 Jun;39(13):1147-1158.

11. Flexural strength and porosity of cements. Birchall J, Howard AJ, Kendall K. Nature. 1981 Jan;289:388-390

12. Bye GC (1999). Portland Cement, 2ndedition: Composition, Production and Properties. United Kingdom:Thomas Telford Ltd.248 p.

13. Placement of mineral trioxide aggregate using two different techniques. Aminoshariae A, Hartwell GR, Moon PC. J Endod. 2003 Oct;29(10):67982. PMID: 14606796.

14. Comparative chemical study of MTA and Portland cements. Oliveira MG, Xavier CB, Demarco FF, Pinheiro AL, Costa AT, Pozza DH. Braz Dent J. 2007 18(1):3-7. PMID: 17639192.

15. Microstructure and strength of hydrated cement.Feldman RF Beaudoin JJ. CemConcr Res. 1976 May;6(3):398-400.

16. Mehta PK (1993). Concrete: Structure, Properties, and Materials. New York: Prentice Hall. 704 p.

17. Relationship between pore structure and mechanical properties of ordinary concrete under bending fatigue.Zhang B. Cem and Concr Res. 1998 May;28(5):699-711.

18. Compressive strength and pore structure of high-performance concrete after exposure to high temperature up to $800 \mathrm{C}$.Chan $\mathrm{Y}$, Luo $\mathrm{X}$, Sun W. CemConcr Res. 2000 30(2):247251.

19. Asthana R, Kumar A, Dahotre N (2006). Materials Processing andManufacturing Science, First Edition. United Kingdom: ButterworthHeinemann. 656 p.

20. Aligizaki KK (2005). Pore Structure of Cement-Based Materials: Testing, Interpretation and Requirements. Boca Raton: CRC Press, LLC. 432 p.

21. Determination of pore size distribution and surface area of several materials using mercury porosimetry and gas adsorption. Tian Y, Chen Kefu. China Pulp and Paper. 2004-04.
22. The Characterization of Porous Solids from Gas Adsorption Measurements.Lee CK, Chiang AST, Tsay CS. Key Eng Mater. 1996 115:21-44.

23. Ultrasonic debridement of root canals: acoustic cavitation and its relevance. 1988. Ahmad M, Pitt Ford TR, Crum LA, Walton AJ. IntEndod J. 2009 May;42(5):391-8. PMID: 19356172 